\title{
Association Between Third-Generation Fluoroquinolones and Achilles Tendon Rupture: A Self-Controlled Case Series Analysis
}

\author{
Takasbi Chinen, $M D^{1,2}$ \\ Yusuke Sasabucbi, MD, MPH, $P b D^{2}$ \\ Hiroki Matsui, MPH ${ }^{2,3}$ \\ Hideo Yasunaga, $M D, P b D^{2,3}$ \\ 'Department of Clinical Oncology, Jichi \\ Medical University, Tochigi, Japan \\ ${ }^{2}$ Data Science Center, Jichi Medical \\ University, Tochigi, Japan \\ ${ }^{3}$ Department of Clinical Epidemiology \\ and Health Economics, School of Public \\ Health, The University of Tokyo, Tokyo, \\ Japan
}

\begin{abstract}
PURPOSE We investigated whether use of third-generation fluoroquinolones is associated with occurrence of Achilles tendon rupture using a case series analysis in which patients served as their own control.
\end{abstract}

METHODS We used administrative claims data to identify residents in a single Japanese prefecture who were enrolled in National Health Insurance and Elderly Health Insurance from April 2012 to March 2017 and experienced Achilles tendon rupture after receiving an antibiotic prescription. Antibiotics were categorized into 3 groups: first- and second-generation fluoroquinolones, third-generation fluoroquinolones, and nonfluoroquinolones. We used a conditional Poisson regression model to estimate the incidence rate ratio (IRR) of Achilles tendon rupture during the antibiotic exposure period relative to the nonexposure period for each patient. An exposure period was defined as 30 days from start of the prescription.

RESULTS Analyses were based on 504 patients with Achilles tendon rupture who had received antibiotic prescriptions. Risk of rupture was not significantly elevated during exposure to third-generation fluoroquinolones (IRR $=1.05 ; 95 \% \mathrm{Cl}$, $0.33-3.37$ ) and nonfluoroquinolones (IRR $=1.08 ; 95 \% \mathrm{Cl}, 0.80-1.47$ ). In contrast, risk was significantly elevated during exposure to first- and second-generation fluoroquinolones (IRR $=2.94 ; 95 \% \mathrm{Cl}, 1.90-4.54)$. Findings were similar across subgroups stratified by sex and by recent corticosteroid use.

CONCLUSIONS Our analysis showed that third-generation fluoroquinolone use was not associated with an increased risk of Achilles tendon rupture. These antibiotics may be a safer option for patients in whom this risk is elevated, such as athletes.

Ann Fam Med 2021;19:212-216. https://doi.org/10.1370/afm.2673.

\section{INTRODUCTION}

$\mathrm{F}$ luoroquinolone-induced tendon injury is a rare event. Nevertheless, a systematic review found that exposure to fluoroquinolones was associated with an increased occurrence of Achilles tendon rupture. ${ }^{1}$ In a population-based cohort study, 5 patients experienced Achilles tendon rupture among 28,262 fluoroquinolone users (standardized incidence rate ratio $[\mathrm{IRR}]=3.1 ; 95 \% \mathrm{CI}, 1.0-7.3) .{ }^{2} \mathrm{~A}$ case-crossover study found that the odds of rupture were doubled with fluoroquinolone exposure relative to nonexposure (odds ratio $=2.0 ; 95 \% \mathrm{CI}, 1.2-3.3$ ). ${ }^{3}$ In a case-control study that adjusted for potential confounders, risk was more than quadrupled (adjusted odds ratio $=4.3 ; 95 \% \mathrm{CI}, 2.4-7.8$ ). ${ }^{4}$ Several studies, however, have indicated that the risk of Achilles tendon rupture varies depending on the type of fluoroquinolone. ${ }^{1,4}$

Newer, third-generation fluoroquinolones such as garenoxacin and sitafloxacin developed in 2006 and 2008, respectively, have been widely used in recent years. ${ }^{5,6}$ Previous studies have investigated the associations between first- and second-generation fluoroquinolones and Achilles tendon rupture ${ }^{6,7} ;$ however, the association for third-generation fluoroquinolones remains unclear. Several studies were unable to successfully control for 
interindividual confounding factors, including age, sex, renal failure, and corticosteroids use. ${ }^{1,3}$ Self-controlled case series analysis can address this shortcoming by eliminating time-invariant intra-individual confounders, and comparing incidence of an outcome during an exposure period relative to a nonexposure period. ${ }^{8,9}$

The aim of the present study was to investigate the association between fluoroquinolones and Achilles tendon rupture, particularly focusing on third-generation fluoroquinolones, by self-controlled case series analysis.

\section{METHODS}

\section{Data Source and Study Population}

All people in Japan are registered with 3 health insurance systems: employees aged $<75$ years and their families with Employee Health Insurance; nonemployees aged $<75$ years and their families with National Health Insurance; and adults aged 75 years and older with Elderly Health Insurance. ${ }^{10,11}$ The insurers for the first system are employers, while the insurers for second and third systems are municipalities. For this study, we used administrative claims data based on the second and third systems that were obtained from municipalities within the Kumamoto Prefecture. At the time of the study, the database contained approximately 780,000 enrollees and represented about $44 \%$ of residents in the prefecture. ${ }^{10,11}$ The database included the following demographic, medical, and pharmacy claims data: patient sex and birth month, date-stamped diagnoses, medical procedures, medications, and anonymized identification numbers assigned to individuals. ${ }^{10} \mathrm{We}$ studied patients in the database who experienced Achilles tendon rupture during a 5 -year period (April 2012 through March 2017) and were prescribed an antibiotic at some time during that period.

The Institutional Review Board of Jichi Medical University approved the study (Number 17-002), and informed consent was waived because of the anonymous nature of the data.

\section{Self-Controlled Case Series Analysis}

Self-controlled case series analysis aims to estimate the relative incidence for the risk of acute events in a transient short-exposure period compared with other periods using data only for cases (individuals who experience the outcome of interest). ${ }^{9,12}$ The advantage of this analysis is that the effects of time-invariant confounders can be removed by comparing the risk period with other periods for the same person. In this study, we defined the overall IRR for Achilles tendon rupture as the ratio of the incidence rate in the exposure period compared with that in the nonexposure period, ${ }^{12,13}$ as described subsequently.

\section{Periods}

The observation period for a given patient was defined as the period from the first month to the last month of

\section{Figure 1. Schematic diagram of the observation period for a hypothetical patient according to the self- controlled case series design.}

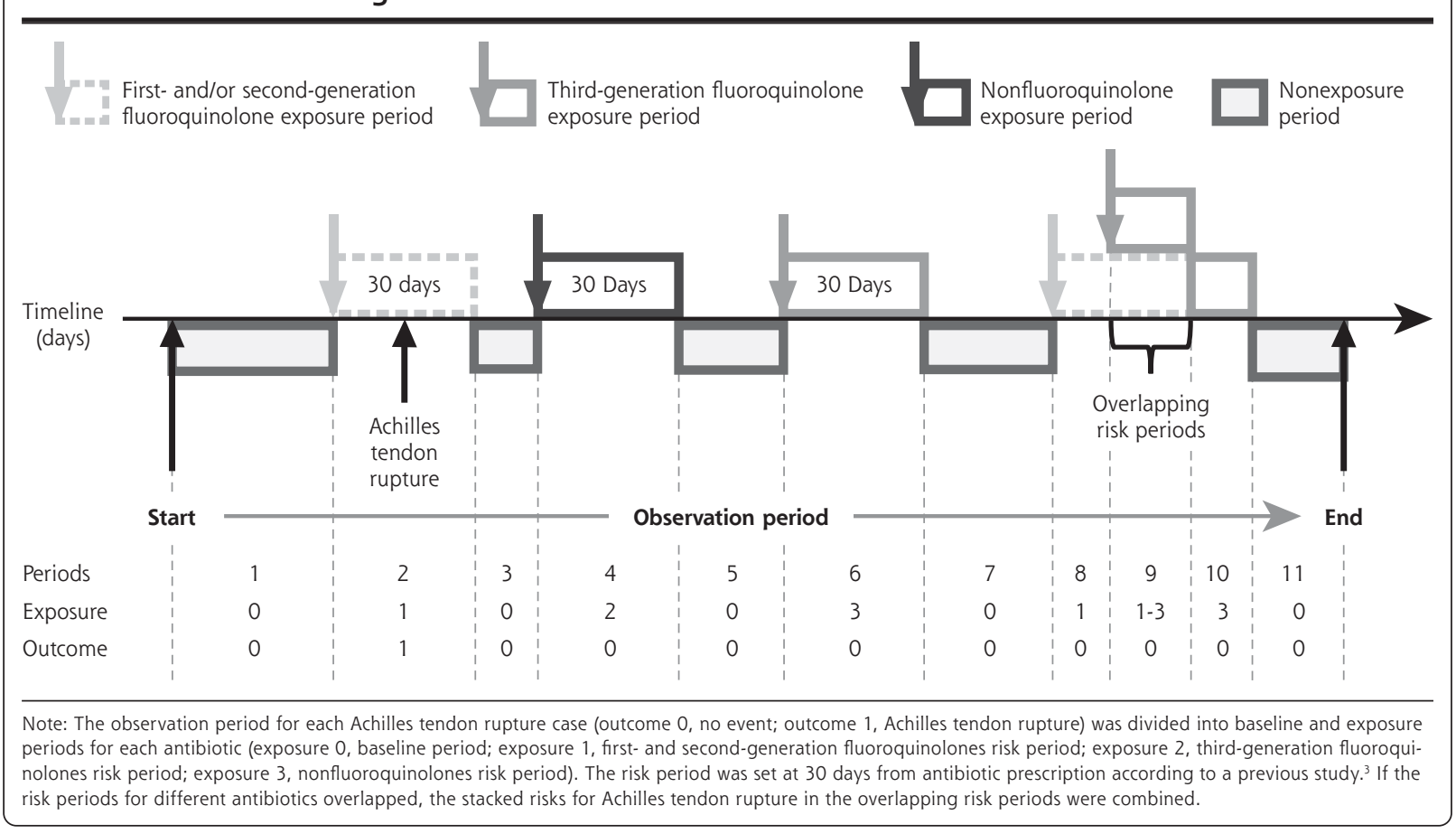


his or her insurance coverage (Figure 1). We divided the observation period into antibiotic exposure and nonexposure periods.

The exposure period (risk period) was defined as the 30-day period starting on the date of an intravenous or oral antibiotic prescription (Figure 1), based on a previous case-crossover study. ${ }^{3}$ In Japan, intravenous and oral antibiotics can be prescribed in both hospitals and clinics, and we therefore extracted all antibiotic prescriptions from the database. Eye drops and ointments were not included in antibiotic exposure. We categorized antibiotics into fluoroquinolones and nonfluoroquinolones, and then further classified the fluoroquinolones into 3 generations, as shown in Table $1 .^{5-7}$ If there were overlapping exposure periods for different types of antibiotics, we combined the stacked risks of Achilles tendon rupture in the overlapping exposure periods. ${ }^{14}$

The nonexposure period (baseline period) was the period in which the patient was not prescribed any intravenous or oral antibiotics (Figure 1). We did not define a washout period between exposure and nonexposure periods.

\section{Outcome}

The study outcome was occurrence of Achilles tendon rupture identified from 3 diagnoses encoded by Japanese original claims codes: rupture of the Achilles tendon, partial rupture of the Achilles tendon, and rupture of the musculotendinous junction of the Achilles tendon. We used the date on which the diagnosis was first recorded as the date of rupture.

\section{Statistical Analysis}

Data are presented as numbers and percentages for categorical variables, and as medians with interquartile ranges for continuous variables. The overall incidence rate of Achilles tendon rupture per 100,000 personyears was calculated as the number of new cases of rupture divided by the observation period for the entire population in the database.

For the self-controlled case series analysis, we calculated the IRR and 95\% CI of Achilles tendon rupture with conditional Poisson regression models. We also performed subgroup analyses with patients stratified by sex and by recent corticosteroid use (defined as use in the 30 days preceding Achilles tendon rupture). ${ }^{1,3}$

We referred to previous reports on appropriate use of self-controlled case series analysis ${ }^{15-17}$ to confirm several assumptions made in the present study. Achilles tendon rupture is a rare and abrupt-onset event that does not affect short-term mortality. Use of antibiotics is an intermittent exposure and independent of the event of Achilles tendon rupture. Rupture does not influence subsequent exposure to antibiotics, is independent of consecutive events when recurrent, and does not influence time under observation.

All statistical analyses were conducted using $R$ 3.4.1. software (the R Foundation). The self-controlled case series package was used for that analysis. ${ }^{14}$

\section{RESULTS}

We extracted data for 533 patients who experienced Achilles tendon rupture during the 5-year study period. The median observation period was 4.1 years (interquartile range $=3.7-4.9$ years).

A total of 29 patients did not receive antibiotics during their observation period and were therefore excluded, leaving 504 patients for analyses. Their baseline characteristics are shown in Table 2.

None of the patients experienced more than 1 Achilles tendon rupture. The population incidence rate was 16.0 cases per 100,000 person-years. The total exposure periods during the observation period and the numbers of Achilles tendon ruptures occurring during these exposure periods are shown in Table 3.

The risks of Achilles tendon rupture for the various types of antibiotics are shown in Table 4. Risk was not significantly elevated during exposure to third-generation fluoroquinolones $(\mathrm{IRR}=1.05 ; 95 \% \mathrm{CI}, 0.33-3.37)$

Table 1. Classification of Fluoroquinolones Available in Japan

\begin{tabular}{lll}
\hline $\begin{array}{l}\text { First } \\
\text { Generation }\end{array}$ & $\begin{array}{l}\text { Second } \\
\text { Generation }\end{array}$ & $\begin{array}{l}\text { Third } \\
\text { Generation }\end{array}$ \\
\hline Norfloxacin & Levofloxacin & Garenoxacin \\
Nalidixic acid & Tosufloxacin & Sitafloxacin \\
Pipemidic acid & Ciprofloxacin & Prulifloxacin \\
& Ofloxacin & Moxifloxacin \\
& Lomefloxacin & Pazufloxacin \\
\hline
\end{tabular}

Table 2. Characteristics of Study Patients $(\mathrm{N}=504)$

\begin{tabular}{lc}
\hline Characteristic & Value \\
\hline Age, median (IQR), y & $64(48-74)$ \\
Age-group, No. (\%) & \\
$<20$ years & $28(6)$ \\
$20-60$ years & $170(34)$ \\
$>60$ years & $306(61)$ \\
Sex, No. (\%) & $299(59)$ \\
Male & $205(41)$ \\
Female & \\
\hline IQR $=$ interquartile range. & \\
Notes: Characteristics were as recorded at the start of the observation period.
\end{tabular}


Table 3. Total Exposure Period and Number of Achilles Tendon Ruptures by Antibiotic Type

\begin{tabular}{lcc}
\hline Antibiotic Type & $\begin{array}{c}\text { Total Exposure } \\
\text { Period, Days }\end{array}$ & $\begin{array}{c}\text { No. of Achilles } \\
\text { Tendon Ruptures }\end{array}$ \\
\hline $\begin{array}{l}\text { First- and second-generation } \\
\text { fluoroquinolones }\end{array}$ & 15,000 & 24 \\
Third-generation fluoroquinolones & 4,290 & 3 \\
Nonfluoroquinolones & 64,590 & 29 \\
Overlapping antibiotic types & 7,187 & 3 \\
\hline a During exposure period. & & \\
\hline
\end{tabular}

be avoided for athletes if alternative antibiotics are available. ${ }^{20}$ Taking the results of the present study and previous studies into account, we believe that third-generation fluoroquinolones may be a safer option with respect to risk of Achilles tendon rupture when patients need a fluoroquinolone. Fluoroquinolones are necessary for patients with allergy to $\beta$-lactam antibiotics or with Legionella pneumopbila infection.

Our study has some limitations. First, we used an administrative claims database, in which the accuracy of diagnoses was not validated ${ }_{1}^{10,21}$ and data for patients enrolled in other health insurance systems were not included. In a previous validation study of the Japanese administrative database, however, specificities of 17 diagnoses exceeded $96 \% .{ }^{21}$ We therefore assumed that the diagnosis of Achilles tendon rupture was highly specific. Second, the sample size may have been insufficient to estimate the risk for individual fluoroquinolones. Third, the claims data did not include information on whether the prescribed drugs were actually dispensed. Fourth, the doses used for several fluoroquinolones in Japan are lower than those used in western countries. ${ }^{22}$ For example, the daily dose of oral ciprofloxacin is 0.2 to $0.6 \mathrm{~g}$ in Japan,

\section{Table 4. Incidence Rate Ratios for Achilles Tendon Rupture by Antibiotic Type}

\begin{tabular}{lc}
\hline Antibiotic Type & $\begin{array}{l}\text { Incidence Rate } \\
\text { Ratio (95\% CI) }\end{array}$ \\
\hline $\begin{array}{l}\text { First- and second-generation } \\
\text { fluoroquinolones }\end{array}$ & $2.94(1.90-4.54)$ \\
$\begin{array}{l}\text { Third-generation fluoroquinolones } \\
\text { Nonfluoroquinolones }\end{array}$ & $1.05(0.33-3.37)$ \\
Note: For each patient, comparator was the period with no antibiotic exposure.
\end{tabular}
risk. ${ }^{18}$ Findings of a preclinical study showed that risk of tendon injury was higher with a methylpiperazinyl substituent than with a piperazinyl substituent. ${ }^{19}$ Only a few thirdgeneration fluoroquinolones, such as gatifloxacin and prulifloxacin, have a methylpiperazinyl substituent, whereas most first- and secondgeneration fluoroquinolones do. This difference may explain why the third-generation fluoroquinolones were not associated with Achilles tendon rupture in our study.

Some researchers have argued that use of fluoroquinolones should

Table 5. Subgroup Analyses by Sex and Recent Corticosteroid Use

\begin{tabular}{|c|c|c|c|}
\hline \multirow[b]{2}{*}{ Subgroup } & \multicolumn{3}{|c|}{ Incidence Rate Ratio $(95 \% \mathrm{Cl})$} \\
\hline & $\begin{array}{l}\text { First- and Second- } \\
\text { Generation } \\
\text { Fluoroquinolones }\end{array}$ & $\begin{array}{l}\text { Third-Generation } \\
\text { Fluoroquinolones }\end{array}$ & Nonfluoroquinolones \\
\hline \multicolumn{4}{|l|}{ Sex } \\
\hline Male & $2.94(1.67-5.18)$ & $0.63(0.09-4.60)$ & $1.06(0.71-1.59)$ \\
\hline Female & $2.86(1.45-5.66)$ & $1.67(0.39-7.06)$ & $1.14(0.71-1.83)$ \\
\hline \multicolumn{4}{|c|}{$\begin{array}{l}\text { Recent cortico- } \\
\text { steroid use }^{\mathrm{a}}\end{array}$} \\
\hline Yes & $8.34(2.27-30.67)$ & $6.30(0.61-64.81)$ & $0.92(0.27-3.12)$ \\
\hline No & $2.52(1.32-4.81)$ & $0.00(0.0$ to $\infty)$ & $1.07(0.70-1.64)$ \\
\hline
\end{tabular}


compared with 0.5 to $1.5 \mathrm{~g}$ in western countries. ${ }^{22}$ Fifth, whether repeated exposures to fluoroquinolones incrementally increase the incidence of Achilles tendon rupture remains unknown. Finally, we investigated only Achilles tendon rupture as an outcome and used data from only a single prefecture. Further studies are required to determine whether third-generation fluoroquinolones affect risks of other rare adverse events, such as aortic aneurysm and dissection, ${ }^{23}$ and to perform self-controlled case series analyses using data for the entire Japanese population.

In conclusion, using administrative claims data for a single prefecture in Japan, we found that thirdgeneration fluoroquinolones were not associated with a significant increase in Achilles tendon rupture, whereas first- and second-generation fluoroquinolones were. This information may be useful in personalizing antibiotic therapy for individual patients.

To read or post commentaries in response to this article, go to https://www.AnnFamMed.org/content/19/3/212/tab-e-letters.

Key words: fluoroquinolone; antibiotics; adverse effects; Achilles tendon rupture; tendon injuries; self-controlled case series; precision medicine

Submitted February 6, 2020; submitted, revised, August 19, 2020; accepted September 1, 2020.

Funding support: This work was supported by a Grant-in-Aid for Scientific Research from the Ministry of Education, Culture, Sports, Science and Technology (grant number JP19K19394).

Disclaimer: The views expressed are solely those of the authors and do not necessarily represent official views of the authors' affiliated institutions or funder.

Previous presentation: This work was presented in part in a poster presentation at Society for Clinical Epidemiology Third Annual Meeting; September 28-29, 2019; Kyoto, Japan.

\section{References}

1. Stephenson AL, Wu W, Cortes D, Rochon PA. Tendon injury and fluoroquinolone use: a systematic review. Drug Saf. 2013;36(9): 709-721.

2. Sode J, Obel N, Hallas J, Lassen A. Use of fluroquinolone and risk of Achilles tendon rupture: a population-based cohort study. Eur J Clin Pharmacol. 2007;63(5):499-503.

3. Wise BL, Peloquin C, Choi H, Lane NE, Zhang Y. Impact of age, sex, obesity, and steroid use on quinolone-associated tendon disorders. Am J Med. 2012;125(12):1228.e23-1228.e28.

4. van der Linden PD, Sturkenboom MC, Herings RM, Leufkens HM, Rowlands S, Stricker BH. Increased risk of Achilles tendon rupture with quinolone antibacterial use, especially in elderly patients taking oral corticosteroids. Arch Intern Med. 2003;163(15):1801-1807.

5. Tsutsui A, Yahara K, Shibayama K. Trends and patterns of national antimicrobial consumption in Japan from 2004 to 2016. J Infect Chemother. 2018;24(6):414-421.
6. Adriaenssens N, Coenen S, Versporten A, et al; ESAC Project Group. European Surveillance of Antimicrobial Consumption (ESAC): outpatient quinolone use in Europe (1997-2009). J Antimicrob Chemother. 2011;66(Suppl 6):vi47-vi56.

7. Ball P. Quinolone generations: natural history or natural selection? J Antimicrob Chemother. 2000;46(Suppl T1):17-24.

8. Petersen I, Douglas I, Whitaker H. Self controlled case series methods: an alternative to standard epidemiological study designs. BMJ. 2016;354:i4515.

9. Farrington CP. Relative incidence estimation from case series for vaccine safety evaluation. Biometrics. 1995;51(1):228-235.

10. Hashimoto $H$, Matsui $H$, Sasabuchi $Y$, et al. Antibiotic prescription among outpatients in a prefecture of Japan, 2012-2013: a retrospective claims database study. BMJ Open. 2019;9(4):e026251.

11. Sasabuchi Y, Matsui H, Kotani K, Lefor AK, Yasunaga H. Effect of the 2016 Kumamoto earthquakes on preventable hospital admissions: a retrospective cohort study in Japan. BMJ Open. 2018;8(7): e021294.

12. DiDiodato G, Fruchter L. Antibiotic exposure and risk of communityassociated Clostridium difficile infection: A self-controlled case series analysis. Am J Infect Control. 2019;47(1):9-12.

13. Takeuchi Y, Ando T, Ishiguro C, Uyama Y. Risk of acute asthma attacks associated with nonsteroidal anti-inflammatory drugs: a selfcontrolled case series. Ther Innov Regul Sci. 2017;51(3):332-341.

14. Farrington P, Whitaker H, Weldeselassie YG. Self-Controlled Case Series Studies: A Modeling Guide With R. CRC Press; 2018.

15. Gault N, Castañeda-Sanabria J, De Rycke Y, Guillo S, Foulon S, Tubach F. Self-controlled designs in pharmacoepidemiology involving electronic healthcare databases: a systematic review. BMC Med Res Methodol. 2017;17(1):25.

16. Nordmann S, Biard L, Ravaud P, Esposito-Farèse M, Tubach F. Caseonly designs in pharmacoepidemiology: a systematic review. PLoS One. 2012;7(11):e49444.

17. Whitaker HJ, Steer CD, Farrington CP. Self-controlled case series studies: Just how rare does a rare non-recurrent outcome need to be? Biom J. 2018;60(6):1110-1120.

18. Bisaccia DR, Aicale R, Tarantino D, Peretti GM, Maffulli N. Biological and chemical changes in fluoroquinolone-associated tendinopathies: a systematic review. Br Med Bull. 2019;130(1):39-49.

19. Kashida Y, Kato M. Characterization of fluoroquinolone-induced Achilles tendon toxicity in rats: comparison of toxicities of 10 fluoroquinolones and effects of anti-inflammatory compounds. Antimicrob Agents Chemother. 1997;41(11):2389-2393.

20. Lewis T, Cook J. Fluoroquinolones and tendinopathy: a guide for athletes and sports clinicians and a systematic review of the literature. J Athl Train. 2014;49(3):422-427.

21. Yamana H, Moriwaki M, Horiguchi H, Kodan M, Fushimi K, Yasunaga $\mathrm{H}$. Validity of diagnoses, procedures, and laboratory data in Japanese administrative data. J Epidemiol. 2017;27(10):476-482.

22. Hori K, Yamakawa K, Yoshida N, Ohnishi K, Kawakami J. Detection of fluoroquinolone-induced tendon disorders using a hospital database in Japan. Pharmacoepidemiol Drug Saf. 2012;21(8):886-889.

23. Pasternak B, Inghammar M, Svanström H. Fluoroquinolone use and risk of aortic aneurysm and dissection: nationwide cohort study. BMJ. 2018;360:k678. 